\title{
Isolated effects of regional hyperthermic perfusion on the development of peritoneal metastasis and overall survival in rats with transplanted high-grade ovarian carcinoma
}

Iaroslav Gennadevich Murazov, Anna Sergeevna Artemyeva², Anna Olegovna Niuganen², Konstantin Yurjevich Senchik ${ }^{3}$

${ }^{I}$ Scientific Laboratory of Cancer Chemoprevention and Oncopharmacology, FSBI «N.N. Petrov National Medical Research Center of Oncology» of the Ministry of Healthcare of the Russian Federation.

${ }^{2}$ Department of Pathology, FSBI "N.N. Petrov National Medical Research Center of Oncology" of the Ministry of Healthcare of the Russian Federation.

${ }^{3}$ Department of Soft Tissue and Bone Tumors, FSBI «N.N. Petrov National Medical Research Center of Oncology» of the Ministry of Healthcare of the Russian Federation.

\section{A BSTRACT}

Aim: To construct a perfusion circuit for experimental open hyperthermic intraperitoneal perfusion (HIPEP) and to evaluate the antitumor effects of regional hyperthermia in a model of advanced syngeneic high-grade ovarian carcinoma in vivo.

Method: 24 mature female Wistar rats underwent intraperitoneal transplantation of ascitic ovarian tumor $1 \times 10^{7}$ cells per rat. 48 hours after transplantation the animals were randomized into two groups: I. NIPEP group (12 rats) - normothermic intraperitoneal perfusion (NIPEP) with normal saline at room temperature during 45 minutes; II. HIPEP group (12 rats) - open hyperthermic intraperitoneal perfusion (HIPEP) with normal saline $\left(40.5-41.5^{\circ} \mathrm{C}\right)$ during 45 minutes. Endpoints included overall survival (OS), the total peritoneal cancer index (PCI), ascites weight and the grade of ascites hemorrhage.

Results: In both groups all animals survived the procedure, in the HIPEP group one rat died due to infectious complications on day 32. Compared with NIPEP group HIPEP with normal saline significantly increased the median OS from 19 to 39 days (log-rank test, $\mathrm{P}=0.0013$ ), reducing the risk of death by $68 \%$ (HR=0.32; $95 \%$ CI 0.13-0.82). The open HIPEP without a cytostatic was associated with significantly lower total PCI (14 vs 5 points, $\mathrm{P}=0.0155)$. In the HIPEP group 3 of 12 animals had intrathoracic tumor spread with malignant pleural effusion without signs of peritoneal carcinomatosis and ascites.

Conclusion: The transplanted syngeneic tumor is a valid model that allows to quantitatively assess antitumor activity of intraperitoneal perfusion therapy. Our preclinical data confirmed the role of regional hyperthermia in the treatment of peritoneal carcinomatosis in ovarian tumors.

Key words: Ovarian cancer, peritoneal carcinomatosis, hyperthermia, peritoneal cancer index, HIPEC, preclinical study.

$\triangle$ Dr. Iaroslav Gennadevich Murazov,

Scientific Laboratory of Cancer Chemoprevention and Oncopharmacology, FSBI «N.N. Petrov National Medical Research Center of Oncology» of the Ministry of Healthcare of the Russian Federation, SaintPetersburg, Russian Federation, 68, Leningradskaya str., Pesochniy Settlement, Saint-Petersburg, 197758, Russian Federation.

E-mail: yaroslav84@yandex.ru

Received: 2021-04-07 / Revisions: 2021-04-21

Accepted: 2021-04-23 / Published online: 2021-07-01 


\section{Introduction}

Peritoneal surface malignancy may occur as a primary disease or as a metastatic tumor originating most often from gastrointestinal tract and gynecological cancers. Peritoneal carcinomatosis (PC) is an unfavorable prognostic factor associated with rapid disease progression, significant deterioration in the quality of life and high mortality of patients [1]. Thus, the 5-year OS of patients with epithelial ovarian cancer (EOC) and PC is about 29\% [2]. Unfortunately, systemic chemotherapy has low efficacy against tumor deposits that have spread along the peritoneum, due to poor blood supply and the low ability of cytostatics to penetrate into intraperitoneal tumor nodules [3]. PC should be considered as a local disease that requires special approaches to treatment. The well-known synergism of the action of regional hyperthermia (40.5-43 $\left.{ }^{\circ} \mathrm{C}\right)$ and chemotherapeutic agents has become the reason for a wide study and introduction into clinical practice of hyperthermic intraperitoneal chemotherapy (HIPEC) [4]. Technically HIPEC can be performed using an open, closed, semi-open, laparoscopic technique or using an expander. Hyperthermia not only enhances the antitumor activity of cytostatics, but also has antitumor activity itself. The direct cytostatic and cytotoxic effects of supranormal temperatures begin at $40-41{ }^{\circ} \mathrm{C}$, while the synergism between hyperthermia and cytostatics begins at $39{ }^{\circ} \mathrm{C}$ and decreases at temperature above $43{ }^{\circ} \mathrm{C}$. Temperature above $44{ }^{\circ} \mathrm{C}$ induce apoptosis in normal cells $[3,5]$. Currently, HIPEC is widely used in the treatment of PC in pseudomyxoma peritonei and peritoneal mesothelioma, peritoneal metastases of colorectal cancer. At the same time, for primary and recurrent EOC with PC the use of HIPEC is not recommended outside clinical trials $[6,7]$. This is due to the lack of standardization of the HIPEC procedure (technique, antineoplastic drugs and their doses, carrier solutions, optimal temperature and duration of perfusion, flow rate of perfusate etc.), as well as the absence of developed algorithm for managing adverse events associated with the procedure. Moreover, current clinical data failed to isolate the curative effects of hyperthermia itself. Until more data are available from evidence-based studies, it is reasonable to conclude that a strategy of surgical cytoreduction and HIPEC is rational and interesting, though still an investigative approach in the management of advanced-stage epithelial ovarian cancer.

A critical stage in the investigation and standardization of perfusion technologies for the treatment of PC are preclinical studies on valid models in vivo. Commonly two types of EOC models are used for PC development in rodents: (1) cell lines and patient-derived xenografts of human tumors; (2) syngeneic models. In order to obtain intraperitoneal dissemination, the tumor can be transplanted intraperitoneally or orthotopically (under the ovarian bursa membrane) [8]. The use of immunodeficient animals for transplantation of human xenografts does not allow studying the interaction of a tumor with microenvironment in the context of the development of an immune response, inflammation, vascularization, and interaction with stromal components. Syngeneic models, on the contrary, make it possible to study the development of the antitumor immune response, epithelial-stromal interactions, and tumor vascularization. The use of immunocompetent animals provides a low incidence of infectious complications in comparison with immunodeficient animals. It should be noted that the hardware used for the experimental HIPEC is quite diverse. Each perfusion circuit is in fact a unique 
multicomponent system [9-12]. Here we used an original peritoneal perfusion system, constructed from domestic devices.

The aim of our study is to assess the antitumor effect of open hyperthermic intraperitoneal perfusion (HIPEP) with normal saline (40.5$41.5^{\circ} \mathrm{C}$ ) on the development of $\mathrm{PC}$ and $\mathrm{OS}$ in female Wistar rats with syngeneic high-grade ovarian carcinoma.

\section{Materials and methods}

Experimental design

A single intervention proceeded $48 \mathrm{~h}$ after intraperitoneal (IP) tumor transplantation. Animals were randomized into two groups: I. NIPEP group (12 rats) - normothermic intraperitoneal perfusion (NIPEP) with normal saline at room temperature during 45 minutes; II. HIPEP group $(n=12)$ - peritoneal cavity of the rats was perfused with heated normal saline according to "Coliseum" (open) technique (parameters are described below).

\section{Animals}

The study was approved by the local ethics committee (REC No 3/297-20) and carried out in accordance with the European Convention for the Protection of Vertebrate Animals used for Experimental and Other Scientific Purposes: Appendix A of the ETS 123 and Directive 2010/63/EU on the protection of animals used for scientific purposes. Twenty four outbred female mature Wistar rats with median weight $294 \mathrm{~g}$ (min-max 274-381 g) were purchased from "Rappolovo" animal nursery (Leningrad Region, Russia). Rats were housed in polypropylene cages in a conventional facility (temperature $20-24^{\circ} \mathrm{C}$, relative humidity $50-60 \%, 12 \mathrm{~h}$ light/12 h dark cycle). Animals received standard laboratory crop (Moscow, Russia) and tap water ad libitum.

\section{The ovarian cancer rat model}

The tumor strain was obtained as a result of transplacental carcinogenesis using high doses of 7,12-dimethylbenzanthracene (DMBA). The initial histological type of tumor is metastatic papillary adenocarcinoma, at present it is an ascitic tumor [13]. Tumor strain is maintained in female Wistar rats. On days 7-9 after IP inoculation, ascitic fluid was collected and transplanted IP to experimental animals $\left(1 \times 10^{7}\right.$ cells in $0.5 \mathrm{ml}$ of normal saline per rat).

Open intraperitoneal perfusion technique Scheme of the experimental perfusion circuit is shown in Figure 1. Thirty minutes prior to and then twice a day on days 0-2 postoperatively, meloxicam («Renewal», Novosibirsk, Russia) was given subcutaneously $2 \mathrm{mg} / \mathrm{kg}$ for analgesia. Rats were anesthetized using isoflurane (Abbott Laboratories Limited, Queenborough, UK), 5\% for induction and 2\% for maintenance. No warming mattress was used during HIPEP, to avoid systemic hyperthermia. A midline laparotomy was performed with a 6-8 cm incision. A "Coliseum" was formed by hemming the edges of the abdominal wall with a surgical suture to a metal ring on a tripod. The inflow line was placed in the left subdiaphragmatic space and the outflow line in the right paracolic gutter. The parameters of the perfusion system were as follows: volume of perfusate (normal saline, "Slavyanskaya Apteka" LLC, Vladimir region, Russia) - $210 \mathrm{ml}$, flow rate - $30 \mathrm{ml} / \mathrm{min}$, abdominal cavity temperature $-40.5-41.5^{\circ} \mathrm{C}$ (for HIPEP group), perfusion time - 45 minutes. During perfusion the abdomen was massaged gently to achieve a uniform heat distribution. After completion of the perfusion, the abdominal wall was closed using 3/0 polyglactin sutures for the muscular layer and $3 / 0$ non-absorbable polyester sutures for the skin. Additionally, the wound was strengthened 


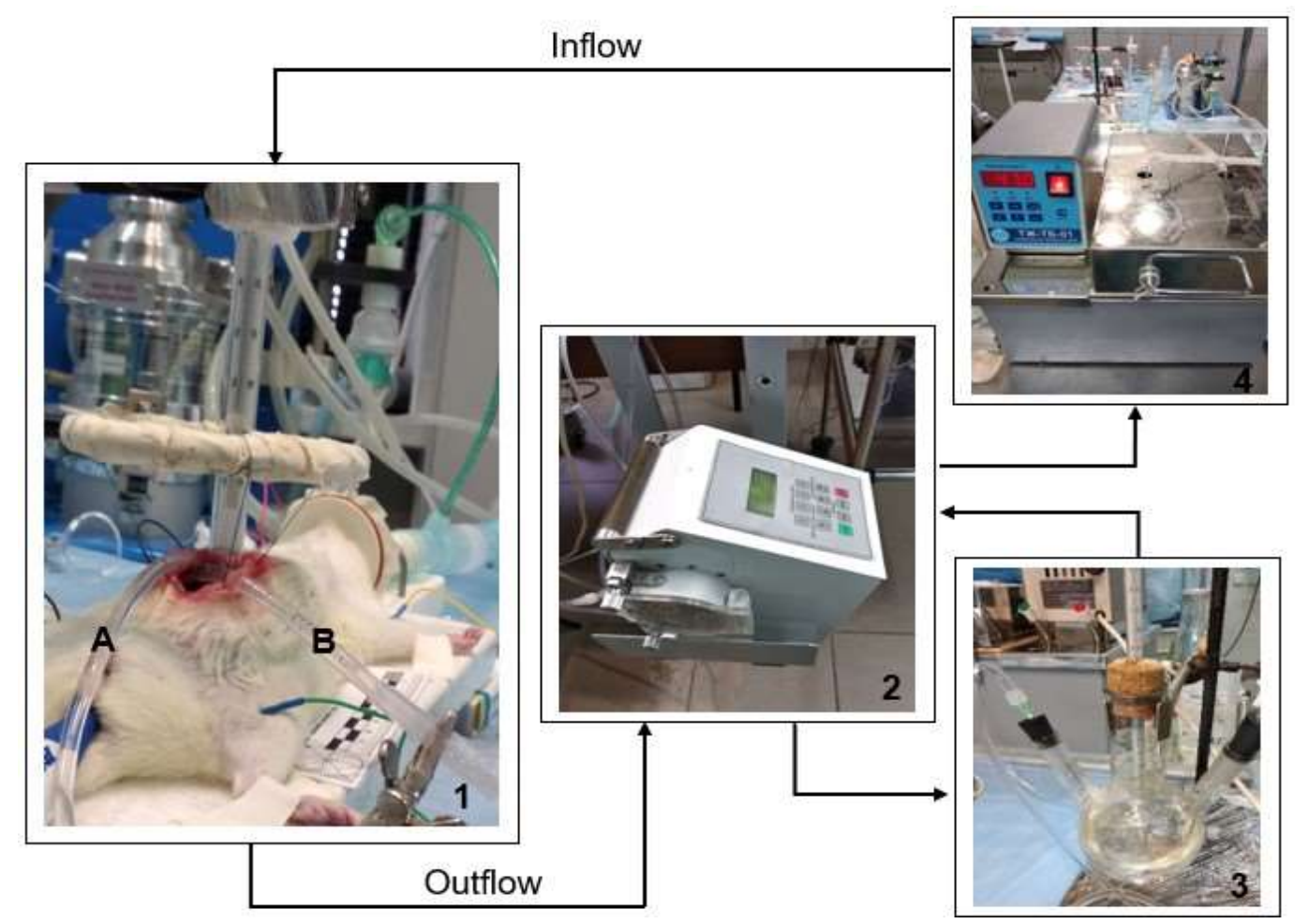

Figure 1. Scheme of the perfusion circuit for an experimental open NIPEP and HIPEP. 1 - position of the animal during the procedure: (A) - inflow line, (B) - outflow line, diameter $4 \mathrm{~mm}$; 2 - perfusion roller pump (Russian State Scientific Center for Robotics and Technical Cybernetics, Saint-Petersburg, Russia); 3 reservoir for perfusate; 4 - thermostatic water bath (Russian State Scientific Center for Robotics and Technical Cybernetics, Saint-Petersburg, Russia).

with BF-6 glue (JSC «Werteks», SaintPetersburg, Russia). Immediately after wound closure rats were given with $10 \mathrm{ml}$ saline subcutaneously for rehydration. Cefepime (JSC «Sintez», Kurgan, Russia) $60 \mathrm{mg} / \mathrm{kg}$ was injected intramuscularly after perfusion and then for next 4 days once daily. The animal was placed on the absorbent sheet in a plastic cage. A heating platform (set at $40^{\circ} \mathrm{C}$ ) was placed under the half of the cage in order to prevent hypothermia. To reduce stress, medium enrichment agents (minks) were placed into the cage. The first five days the postoperative wound was treated with Levomekol ointment (JSC "Nizhfarm", Nizhny Novgorod, Russia).

Follow-up. The animals were observed throughout the entire period of their life. OS was defined as time from the day of tumor transplantation to day of animal death. The total peritoneal cancer index (PCI), ascites weight and its grade of haemorrhagia were determined at autopsy. The total PCI was determined according to the modified method of Y.L. Klaver. et al. [14]. After laparotomy, the abdomen was carefully examined for tumor growth at eight different sites (see Table 1). The tumor load at each site was scored semi quantitatively: 0 - no macroscopic tumor; 1 limited tumor growth (diameter 1-2 $\mathrm{mm}$ ); 2 moderate tumor growth (diameter $2-4 \mathrm{~mm}$ ); 3 - abundant (diameter more than $4 \mathrm{~mm}$ ). In the case of multiple nodes in the organ, the node with the largest diameter was selected. The sum of scores from all sites represented the total PCI for that animal. The grade of ascites haemorrhagia was determined using a semi quantitative visual scale, where 0 - absence of hemorrhagic ascites; 1 - weak hemorrhagic ascites; 2- moderate hemorrhagic ascites; 3 strong hemorrhagic ascites. Organs of the 
reproductive system of all animals, as well as organs and tissues with macroscopic signs of PC, were subjected to standard histological examination by light microscopy after hematoxylin and eosin staining. Cytological smears of ascites and pleural effusion were examined after Pappenheim's stain.

\section{Statistical Analysis}

Statistical analysis was performed using GraphPad Prism version 8.0. Based on pilot preclinical study (data was not published) required sample size was 12 animals per group to reject the null hypothesis that survival in the NIPEP group and the HIPEP group are equal with power $(1-\beta)=80 \%$ and $\alpha=0.05$ [15]. Nonparametric testing for the continuous data was performed using Mann-Whitney test. Comparison of categorical values was done using Fisher's exact test. All tests were twosided; the level of statistical significance was set at a $\mathrm{P}$ value of $<0.05$. Survival outcomes were analyzed and expressed using KaplanMeier curves and compared with the log-rank test. The Cox proportional-hazards model was used to obtain hazard ratio (HR) and its 95\% confidence interval (95\% CI).

\section{Results}

All animals in the NIPEP and HIPEP groups survived the procedure. On day 32 after HIPEP one of 12 rats died from infectious complications. Mean anesthesia time was 115 minutes (min-max: 100-142). The dynamics of intraperitoneal and rectal temperature during HIPEP is shown in Figure 2. The set temperature in the abdominal cavity was reached 10-17 minutes after the installation of the lines and the start-up of the circuit.

After IP transplantation tumor take rate achieved nearly $100 \%$ and led to haemorrhagic ascites formation and PC. Cancer-related mortality in rats was nearly $100 \%$. The autopsy revealed tumor lesions in mesentery, greater omentum, parietal peritoneum and diaphragm. High volume tumor implants were observed in ovaries, horns and body of uterus, perigonadal fat pads (Figure 3).

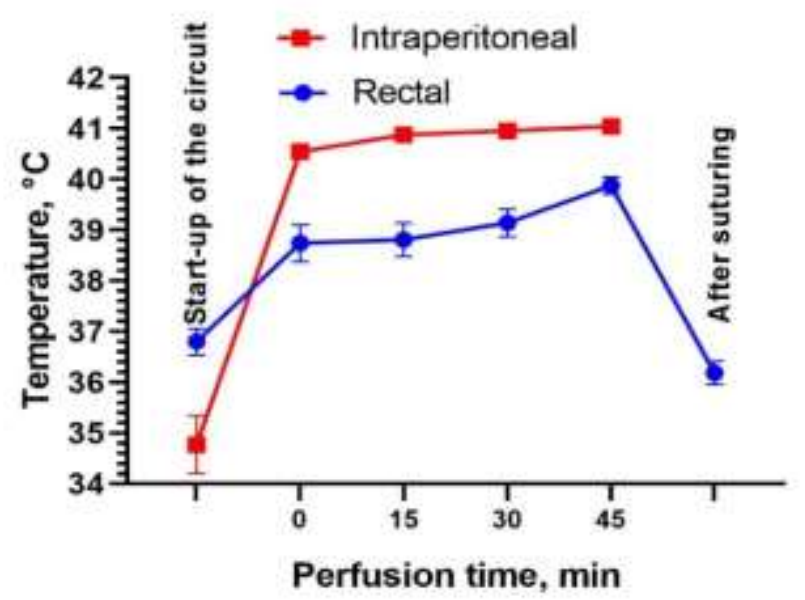

Figure 2. The temperature course during HIPEP in the abdomen and in the rectal cavity.

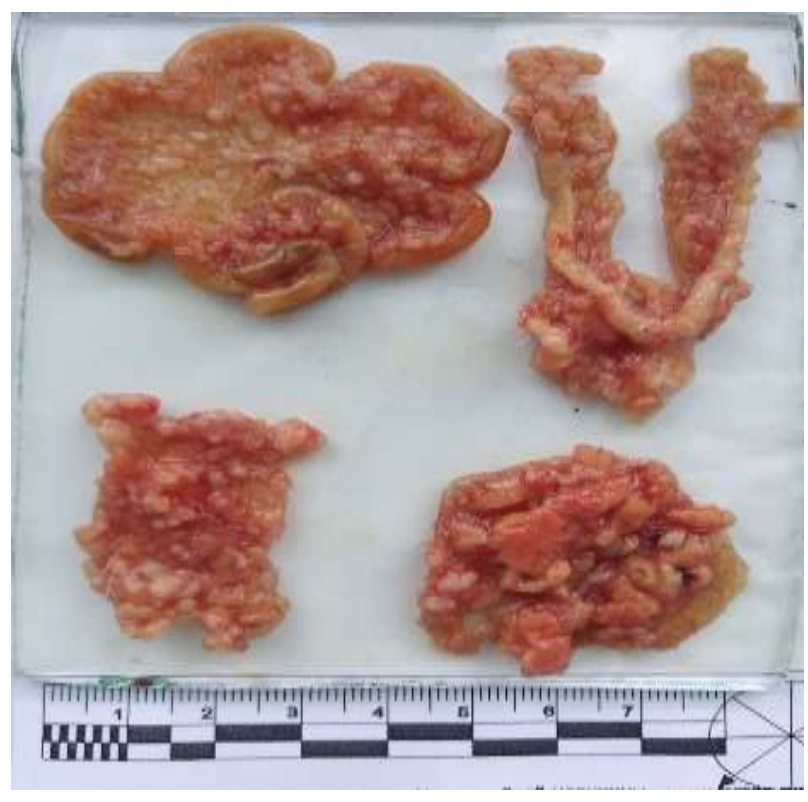

Figure 3. Macroscopic tumor view in rat with transplanted syngeneic ovarian carcinoma (NIPEP group). Tumor implants in mesentery, uterine horns and body with perigonadal fat pads, greater omentum and diaphragm.

The development of peritoneal metastasis was accompanied by hemorrhagic ascites. In smears 
of ascitic fluid extensive focuses of high-grade serous carcinoma discretely lying among the fields of cells of the monocytic series were determined. Cells were polymorphic, sharply atypical with large nuclei with nucleoli and a high nuclear-cytoplasmic ratio (Figure 4).

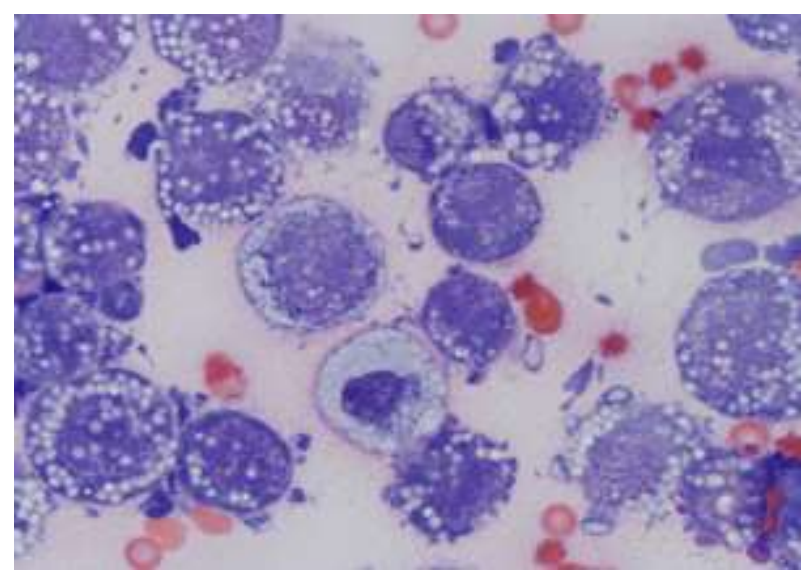

Figure 4. Smear of ascitic fluid, Pappenheim's stain, $\times 1000$.
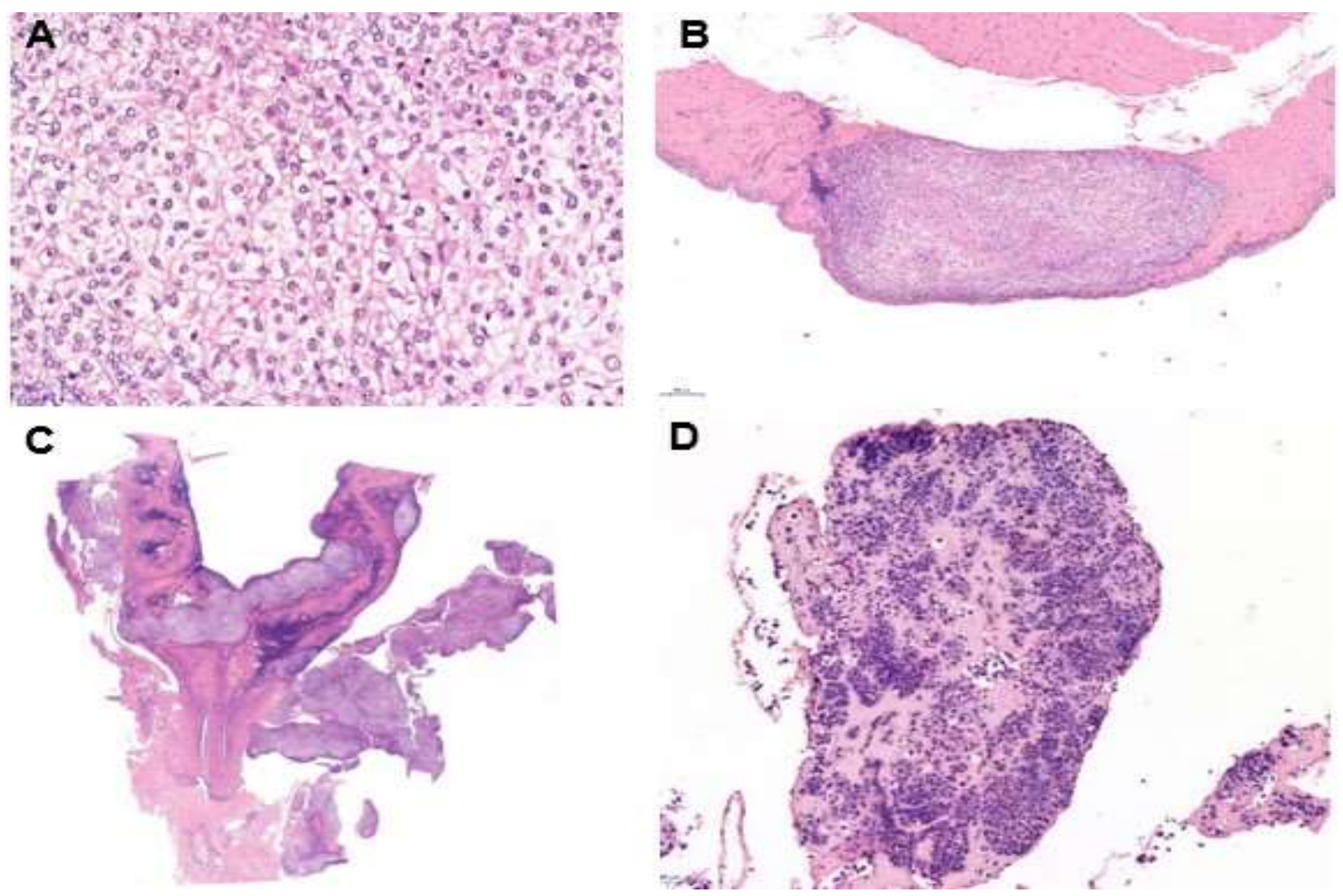

Figure 5. Microscopic tumor view. (A) - focus of serous high-grade carcinoma, haematoxylin and eosin, $\times 400$; (B) - tumor nodule on the parietal peritoneum, haematoxylin and eosin, $\times 60$; (C) - invasion of the tumor into the body of the uterus and uterine horns, haematoxylin and eosin, $\times 5$; (D) - a fragment of the greater omentum with tumor infiltration, haematoxylin and eosin, $\times 40$. 
difference in survival outcome was significant, log-rank test $P=0.0013 ; H R=0.32 \quad(95 \% C I$ 0.13-0.82). One rat from the HIPEP group was sacrificed on day 138 after tumor transplantation without any signs of tumor growth (confirmed on autopsy).

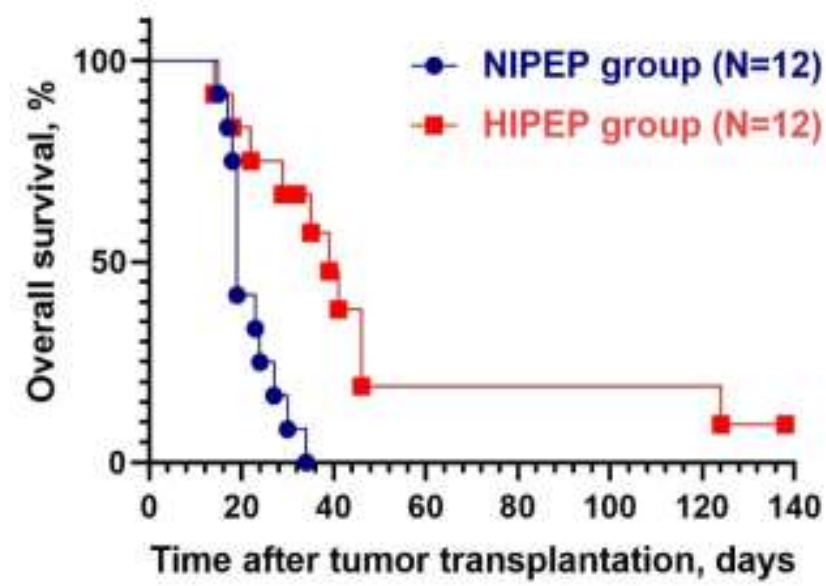

Figure 6. Kaplan-Meier analysis of survival curves of rats with transplanted ovarian carcinoma. NIPEP - normothermic intraperitoneal perfusion with normal saline; HIPEP - hyperthermic intraperitoneal perfusion with normal saline.

In NIPEP group all animals developed extensive intraperitoneal tumor growth at autopsy. Rats in the HIPEP group were found to have a significantly lower mean of the total PCI than rats in NIPEP group $(P=0.00155)$. There was no significant difference in the ascites weight between groups owing to massive variation of this parameter. Although there was a trend towards a decrease in the HIPEP group. At the same time, ascites from HIPEP group had a more pronounced degree of haemorrhagia. Autopsy findings are shown in Table 1.

\section{Discussion}

The selective effects of hyperthermia on malignant cells as well as its ability to potentiate chemotherapy agents make it a valuable adjunct to intraperitoneal chemotherapy in the management of PC [16]. It is supported by an increasing number of research data. But available clinical data failed to isolate the antitumor effects of hyperthermia itself. Currently, the role of HIPEC in the management of primary and recurrent EOC is still not clear [17]. One of the reasons for this is insufficient experimental data, including preclinical studies on adequate experimental models.

The chosen model of syngeneic ovarian carcinoma in Wistar rats resembles the clinical situation of advanced EOC (stage III-IV) in humans with the PC and ascites. The histological structure corresponded to the most common subtype of human ovarian tumor high-grade serous carcinoma. The model resembles the microscopic disease before the emergence of visible nodules [18].

For our study we designed and tested a new peritoneal perfusion system in tumor bearing rats. We chose to use the "Coliseum" technique for NIPEP and HIPEP due to its advantages over the closed technique. It allows to achieve homogeneous temperature and better control of perfusate distribution within the peritoneal cavity, make the access to the entire cavity easier, prevents of bubble formation in the circuit and organ suction, possibility of debulking of large tumors before perfusion, easier circuit blockage corrections, and minimal risk of thermal damage of organs and tissues [12,19].

Here, we demonstrated that open HIPEP with saline only significantly increased the median survival of the animals from 19 to 39 days (logrank test $P=0.0013$ ) and reduced the risk of death by $68 \%$ (HR for HIPEP group vs NIPEP group 0.32; 95\% CI 0.13-0.82).

The exact mechanism of antitumor activity of hyperthermia is not fully understood. It is 
Table 1. Influence of HIPEP with normal saline on the spread of transplanted ovarian carcinoma and the ascites weight on autopsy.

\begin{tabular}{|c|c|c|c|}
\hline Tumor score per site & $\begin{array}{l}\text { NIPEP group } \\
(\mathrm{N}=12)\end{array}$ & $\begin{array}{l}\text { HIPEP group } \\
(\mathrm{N}=12)^{b}\end{array}$ & P value \\
\hline Greater omentum & $3(3-3)$ & $1(0-3)$ & \\
\hline $\begin{array}{l}\text { Space between the liver and } \\
\text { the diaphragm }\end{array}$ & $3(0-3)$ & $0(0-3)$ & \\
\hline Liver hilum & $3(0-3)$ & $0(0-3)$ & \\
\hline Mesentery & $3(0-3)$ & $0(0-3)$ & \\
\hline Diaphragm & $0(0-3)$ & $0(0-3)$ & \\
\hline Ovaries & $1(0-3)$ & $0(0-2)$ & \\
\hline Parietal peritoneum & $0(0-2)$ & $0(0-3)$ & \\
\hline $\begin{array}{l}\text { Uterine horns and body with } \\
\text { perigonadal fat pads }\end{array}$ & $2,5(0-3)$ & $0(0-3)$ & \\
\hline Total PCI & $14(2)$ & $5(2)$ & $\begin{array}{l}\text { Mann Whitney test, } \\
P=0.0155\end{array}$ \\
\hline Ascites weight, $\mathrm{g}^{\mathrm{b}}$ & $\begin{array}{l}67.4 \\
(31.5-100.5)\end{array}$ & $\begin{array}{l}24.6 \\
(11.1-80.1)\end{array}$ & $\begin{array}{l}\text { Mann Whitney test, } \\
P=0.1206\end{array}$ \\
\hline $\begin{array}{l}\text { Grade of ascites } \\
\text { haemorrhagia }^{b}\end{array}$ & $2(2-2)$ & $3(2-3)$ & $\begin{array}{l}\text { Mann Whitney test, } \\
P=0.0379\end{array}$ \\
\hline $\begin{array}{l}\text { Intrathoracic tumor spread } \\
\text { with malignant pleural } \\
\text { effusion, } \mathrm{n}\end{array}$ & 0 & 3 & $\begin{array}{l}\text { Fisher's exact test, } \\
P=0.0932\end{array}$ \\
\hline
\end{tabular}

${ }^{a}$ Values are median (Q1-Q3) and ${ }^{c}$ mean (SEM). ${ }^{b} 3$ animals with malignant pleural effusion and 1 rat with infectious complications were excluded from PCI calculation.

known that the cytotoxic effects of hyperthermia are caused by damage of cell membranes, nuclei, protein denaturation, and changes in the permeability to calcium ions. It disrupts mitotic processes of malignant and non-malignant cells too. In tumor cells, after exposure to hyperthermia, an increase in the number of lysosomes and the activity of lysosomal enzymes is established.

Microcirculation in tumor tissue is suppressed by hyperthermia, which leads to decrease in blood flow to the tumor or its complete stasis, whereas in normal tissues the blood flow increases. These effects, along with a violation of cellular respiration processes, lead to the accumulation of lactic acid and acidification of the tumor microenvironment, which leads to an increase in the number and "fragility" of lysosomes, accompanied by increased death of tumor cells [20].

Noteworthy is the profile of the OS curve in the HIPEP group. The presence of such "tails" may be associated with the activation of antitumor immunity in immunocompetent animals with 
transplanted syngeneic tumor. This profile resembles the OS curve of patients receiving checkpoint inhibitors with a long-term response to this therapy [21]. It is known that in addition to the direct cytotoxic effect on tumor cells, hyperthermia stimulates the production of heat shock proteins (HSPs) and their presentation in complex with the major histocompatibility complex class II, activates dendritic cells and natural killers, enhances the adhesive properties of T-cells and their traffic to peripheral tissues and promotes immune surveillance [22].

Locoregional intraperitoneal heating independently contributed to antitumor activity and reduced intraperitoneal tumor dissemination. In the HIPEP group the total PCI was significantly lower and a trend towards a decrease in the ascites weight was noticed. At the same time, ascites in animals from HIPEP group had a more pronounced degree of haemorrhagia. Probably, this was the main cause of death. An interesting observation from the study was that $3 / 12$ rats in HIPEP group had haemorrhagic pleural effusion with malignant cells but without macroscopic evidence of tumor growth in peritoneal cavity and ascites. Such phenomenon may be due to tumor cells migration under conditions of high intraabdominal pressure and supranormal temperature during perfusion.

\section{Conclusions}

In conclusion, the model of syngeneic highgrade ovarian carcinoma in Wistar rats and the original experimental perfusion circuit can be used for further investigation of HIPEC after proper equipment of the operating room by setting general and local ventilation, which will provide a special microclimate during the procedure. Our preclinical results supplemented the data about the antitumor activity of local hyperthermia and its role in the perfusion treatment of PC in ovarian tumors.
Funding: The author(s) received no financial support for the research, authorship, and/or publication of this article.

Conflict of Interest: The authors declare that they have no conflict of interest.

Ethical statement: The study was approved by the local ethics committee (REC No 3/297-20) and carried out in accordance with the European Convention for the Protection of Vertebrate Animals used for Experimental and Other Scientific Purposes: Appendix A of the ETS 123 and Directive 2010/63/EU on the protection of animals used for scientific purposes.

\section{Open Access Statement}

This is an open access journal which means that all content is freely available without charge to the user or his/her institution under the terms of the Creative Commons Attribution NonCommercial License (http://creativecommons.org/licenses/bync/4.0). Users are allowed to read, download, copy, distribute, print, search, or link to the full texts of the articles, without asking prior permission from the publisher or the author.

\section{References}

[1]Helderman RFCPA, Löke DR, Kok HP et al. Variation in Clinical Application of Hyperthermic Intraperitoneal Chemotherapy: A Review. Cancers (Basel). 2019; 11(1): 78.

[2]Reid B.M., Permuth J.B., Sellers T.A. Epidemiology of ovarian cancer: a review. Cancer Biol Med. 2017; 14(1): 9-32.

[3]Goodman MD, McPartland S, Detelich D et al. Chemotherapy for intraperitoneal use: a review of hyperthermic intraperitoneal chemotherapy and early post-operative intraperitoneal chemotherapy. J Gastrointest Oncol. 2016; 7(1): 45-57. 
[4]Sugarbaker PH, Graves T, DeBruijn EA, et al. Early postoperative intraperitoneal chemotherapy as an adjuvant therapy to surgery for peritoneal carcinomatosis from gastrointestinal cancer: pharmacological studies. Cancer Res. 1990; 50(18): 5790-94.

[5]Hager E.D. Intracavitary Hyperthermic Perfusion. In: Madame Curie Bioscience Database [Internet]. Austin (TX): Landes Bioscience 2000-2013, https://www.ncbi.nlm.nih.gov/books/NBK6 059.

[6]Cortez AJ., Tudrej P, Kujawa KA et al. Advances in ovarian cancer therapy. Cancer Chemother Pharmacol. 2018; 81(1): 17-38.

[7]Jewell A, McMahon M, Khabele D. Heated Intraperitoneal Chemotherapy in the Management of Advanced Ovarian Cancer. Cancers (Basel). 2018; 10(9): 296.

[8]Murazov Ia.G., Niuganen A.O., Artemyeva A.S. Experimental modeling of ovarian carcinoma. Laboratory Animals for Science. 2020;

https://doi.org/10.29296/2618723X-202003-05 (In Russian).

[9]Pelz JO, Doerfer J, Hohenberger W et al. A new survival model for hyperthermic intraperitoneal chemotherapy (HIPEC) in tumor-bearing rats in the treatment of peritoneal carcinomatosis. BMC Cancer. 2005; 5:56.

[10]Badrudin D, Sideris L, Perrault-Mercier C, et al. Comparison of open and closed abdomen techniques for the delivery of intraperitoneal pemetrexed using a murine model. J Surg Oncol. 2018; 117(6): 1318-22.

[11]Park EJ, Ahn J, Gwak SW, et al. Pharmacologic Properties of the Carrier Solutions for Hyperthermic Intraperitoneal Chemotherapy: Comparative Analyses Between Water and Lipid Carrier Solutions in the Rat Model. Ann Surg Oncol. 2018; 25(11): 3185-92.

[12] McCabe-Lankford E, Peterson M, McCarthy B. et al. Murine Models of Intraperitoneal Perfusion for Disseminated Colorectal Cancer. J Surg Res. 2019;233:310-22.

[13]Pogosyants EE, Prigozhina EL, Egolina NA. Transplantable rat ovary ascitic tumor. Vopr Oncol 8: 29-36, 1962 (In Russian).

[14] Klaver YL, Hendriks T, Lomme RM, et al. Intraoperative hyperthermic IP CT after CRS cytoreductive surgery for peritoneal carcinomatosis in an experimental model. $\mathrm{Br}$ J Surg. 2010; 97(12): 1874-80.

[15] Schoenfeld DA, Richter JR. Nomograms for calculating the number of patients needed for a clinical trial with survival as an endpoint. Biometrics. 1982;38(1): 163-70.

[16] Sticca RP, Dach BW. Rationale for hyperthermia with intraoperative intraperitoneal chemotherapy agents. Surg Oncol Clin N Am. 2003; 12(3):689-701.

[17] Vergote I, Harter P, Chiva L. Is There a Role for Intraperitoneal Chemotherapy, Including HIPEC, in the Management of Ovarian Cancer? J Clin Oncol. 2019; 37(27): 242023.

[18]Gremonprez F, Willaert W, Ceelen W. Intraperitoneal chemotherapy (IPC) for peritoneal carcinomatosis: review of animal models. J Surg Oncol. 2014; 109(2): 110-16.

[19] González-Moreno S, González-Bayón LA, Ortega-Pérez G. Hyperthermic intraperitoneal chemotherapy: Rationale and technique. World J Gastrointest Oncol. 2010; 2(2):68-75.

[20] Sticca RP, Dach BW. Rationale for hyperthermia with intraoperative intraperitoneal chemotherapy agents. Surg Oncol Clin N Am. 2003; 12(3): 689-701.

[21] Harris SJ., Brown J, Lopez J et al. Immunooncology combinations: raising the tail of 
the survival curve. Cancer Biol Med. 2016; 13(2): 171-93.

[22] Skitzki JJ., Repasky EA, Evans SS. Hyperthermia as an immunotherapy strategy for cancer. Curr Opin Investig Drugs. 2009; 10(6): 550-58. 